\title{
REUNIFICAÇÃO E EVOLUÇÃO RECENTE DOS DESEQUILÍBRIOS REGIONAIS NA ALEMANHA
}

\author{
Prof $^{a}$. Dr ${ }^{\mathrm{a}}$. Barbara-Christine Nentwig Silva \\ Pesquisadora CNPq \\ Pós-graduação em Planejamento Territorial e Desenvolvimento Social/UCSAL e em Geografia/UFBA \\ Avenida Cardeal da Silva, 205 - CEP 40231-902 - Salvador (BA) - Brasil
}

Tel.: (+ 55 71) 32038989 - barbarans@ucsal.br

\section{RESUMO}

Maina Pirajá Silva

mainapiraja@yahoo.com.br

\begin{abstract}
O artigo analisa a evolução, entre 1995 e 2006, dos desequilíbrios regionais na Alemanha, ou seja, menos de um ano após a reunificação alemã de (03/10/1989). O exemplo alemão é bastante expressivo já que integrou a antiga República Democrática Alemã, constituída no modelo comunista, à República Federal da Alemanha, constituída no modelo capitalista. Os desequilíbrios regionais resultam, historicamente, dos processos econômicos, sociais, políticos e culturais que beneficiam determinadas regiões em detrimento de outras. Com base em dados da União Européia (EUROSTAT), foi calculado o índice de Williamson que mede os desequilíbrios regionais. A Alemanha, dividida em Estados, tem baixos índices de desequilíbrios regionais e isto tem sido estável no período analisado. Os desequilíbrios aumentam quando se divide a Alemanha em unidades menores. Destacando a comparação somente com base na renda per capita, a mesma representa, na parte leste, cerca de dois terços da parte oeste e a diferença aumenta bastante quando se analisam os dados por níveis territoriais menores. Assim, a Alemanha tem ainda um grande desafio, o de reduzir estrategicamente seus desequilíbrios regionais em todas as escalas do seu território nacional.
\end{abstract}

Palavras-Chave: Reunificação, desequilíbrios regionais, Alemanha, índice de williamson, PIB per capita.

\begin{abstract}
The paper analyzes the evolution between 1995 and 2006 of the regional imbalances in Germany, i.e. after the German reunification of October 3, 1990. The German example is quite expressive since it joined the former German Democratic Republic, constituted in communist model, to the Federal Republic of Germany, constituted in the capitalist model. Regional imbalances result, historically, of the economic, social, political and cultural processes that benefit some regions over others. Based on data from the European Union (EUROSTAT), we calculated the index that measures Williamson regional imbalances. Germany divided into States has low rates of regional imbalances and this has been stable during the period. Imbalances increase when Germany is divided into smaller units. Highlighting the comparison only on the basis of per capita income, it represents, in the eastern part, about two-thirds of the west. Disparities of income per capita also greatly increase when the data are analyzed by smaller territorial units. Thus, Germany still has a big strategic challenge to reduce its regional imbalances in all scales of their national territory.
\end{abstract}

Key words: Reunification, regional imbalances, Germany, Index of williamson, GDP per capita.

\section{RÉSUMÉ}

L'article analyse l'évolution des déséquilibres régionaux en Allemagne, entre 1995 et 2006, c'est à dire après la réunification allemande de 03/10/1990. L'exemple allemand est particulièrement expressif, car il s'agit de l'intégration de l'ancienne République Démocratique Allemande constituée sur un modèle communiste, à la République Fédérale Allemande, constituée elle sur un modèle capitaliste. Les déséquilibres régionaux résultent, historiquement, des processus économiques, sociaux, politiques et culturels qui bénéficient des régions déterminées aux dépens d'autres régions. Sur la base des données de l'Union Européenne (EUROSTAT), nous avons calculé l'indice de Williamson qui mesure les déséquilibres régionaux. L'Allemagne, divisée en États, présente de faibles indices de déséquilibre régional et cette situation s'est maintenue stable pendant toute la période analysée. Par contre, les déséquilibres augmentent lorsque l'on divise l'Allemagne en unités plus petites. Ainsi, considérant à peine le revenu per capita, on observe dans l'est, des valeurs équivalant aux deux tiers des valeurs du côté ouest et cette différence augmente significativement lorsque l'on analyse les données pour des territoires plus petits. L'Allemagne fait donc face à un grand défi, celui de réduire stratégiquement ses déséquilibres régionaux à toutes les échelles de son territoire national.

Mots- Clés: Réunification, déséquilibres régionaux, Allemagne, indice de williamson, PIB per capita.

\section{INTRODUÇÃO}

O objetivo deste trabalho é o de analisar a evolução recente dos desequilíbrios regionais na Alemanha, certamente o exemplo mais significativo de integração de unidades territoriais constituídas sob a forma de estados nacionais independentes, bastante diferenciados e desequilibrados, ambos colocados no epicentro das complexas questões geopolíticas internacionais. 
A Alemanha, a partir da reunificação entre a parte oriental e ocidental, é também um exemplo dos mais expressivos de busca de rápida eliminação ou expressiva redução das grandes diferenças e desequilíbrios econômicos e sociais (SILVA, 1992). Como reconhece Schmid (1996, p.13) comentando a reunificação: "agora temos a unidade externa - e a igualdade interna? Entre os antigos e novos estados federais existe uma grande diferença econômica e social".

Logo após a reunificação, em 1990, fez parte dos debates públicos a estimativa do tempo necessário para a eliminação ou forte redução das diferenças e desequilíbrios. Os mais otimistas estimavam em torno de uma década e os mais pessimistas em duas ou mais décadas.

Assim, 20 anos após este evento de grande repercussão internacional, é importante realizar um balanço dos desequilíbrios regionais na Alemanha.

\section{CONCEITO BÁSICO, MATERIAIS E MÉTODOS}

Os desequilíbrios regionais podem ser definidos como o resultado "da existência de vantagens, sobretudo econômicas, comparativamente mais favoráveis a determinadas regiões como decorrência de fatores locacionais e de interação, associados a questões econômicas, políticas, sociais e culturais" (SILVA; SILVA; COELHO, 2008, p.16). Estas vantagens são historicamente produzidas e, muitas vezes, chocam pela contundência dos indicadores relacionados com os desequilíbrios regionais, $o$ que provoca a necessidade de formulação de políticas, programas e projetos visando a redução dos mesmos, como aconteceu no Brasil, na segunda metade da década de 50 do século passado, resultando, por exemplo, na criação da Superintendência de Desenvolvimento do Nordeste (SUDENE).

O exemplo recente da Alemanha se enquadra nas considerações acima, com sua experiência única de comparação entre dois países politicamente, socialmente e economicamente bastante diferenciados e desequilibrados que se integram plenamente e que, a partir daí, buscam construir um país mais equilibrado.

Para analisar os desequilíbrios entre a antiga Alemanha Ocidental e a Alemanha Oriental, em diferentes escalas, foram levantadas estatísticas do sistema EUROSTAT, o instituto estatístico da União Européia, para o período 1995 a 2006. Não há dados comparáveis neste banco de dados anteriores a 1995 e o último ano disponível, no momento da elaboração da pesquisa, é o de 2006.

Como a medição dos desequilíbrios foi efetuada com base no índice de desequilíbrio regional de Williamson (1977, original de 1965), os dados levantados foram os seguintes: população nacional, população das regiões, renda per capita nacional e renda per capita das regiões. Quanto mais próximo de zero, menor será o desequilíbrio regional de um determinado país. A vantagem do índice de Williamson é a sua consistência e simplicidade, facilitando sua aplicação e comparação em diferentes contextos e escalas. (SILVA; SILVA; COELHO, 2008)

A equação original de Williamson é a seguinte:

Onde:

fi = população da i-ésima região

$\mathrm{n}=$ população nacional

yi = renda per capita da i-ésima região

$\bar{y}=$ renda per capita nacional

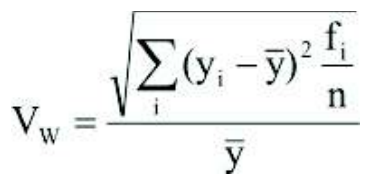




\section{EVOLUÇÃO RECENTE DA ALEMANHA}

Em 2009, a Alemanha comemorou festivamente 20 anos da queda do Muro de Berlin, evento de enorme significado para todos os alemães e para o mundo todo, já que foi o marco inicial do fim da chamada guerra fria com as transformações em todo o leste europeu. No mesmo ano, comemorou-se igualmente os 60 anos da formação constitucional da República Federal da Alemanha (BRD ou RFA), Alemanha Ocidental, criada em 23/05/1949 com o apoio do mundo ocidental. Em 07/10/1949, também foi fundada a República Democrática Alemã (DDR ou RDA), Alemanha Oriental, com o suporte da União Soviética.

Com isto, quatro anos após o fim da Segunda Guerra Mundial, iniciada em 1939 e terminada em 1945, a antiga Alemanha foi dividida em dois estados nacionais, o mesmo ocorrendo com a cidade de Berlin, a capital, fragmentada em Berlin Ocidental e em Berlin Oriental. Em 13/08/1961, com o acirramento da guerra fria, o muro de Berlin é construído, dividindo radicalmente o território da cidade. Com isto, as relações entre as populações das duas partes de Berlin foram praticamente anuladas, o mesmo ocorrendo com os dois estados alemães, com a implantação da chamada cortina de ferro.

No lado oriental, após 1949, ocorreu a implantação do sistema comunista bastante integrado ao bloco liderado pela União Soviética, e, no lado ocidental, teve continuidade o sistema capitalista, bem associado ao bloco liderado pelos Estados Unidos. Por conseguinte, todo o sistema político, econômico, social e cultural era bastante diferenciado nas duas Alemanhas, o mesmo ocorrendo em seu rebatimento espacial, com repercussões regionais e locais muito diversificadas.

No lado ocidental, consolidou-se a chamada economia social de mercado, com resultados significativos, expressos, por exemplo, no chamado milagre alemão e, no lado oriental, a economia socialista, com resultados expressivos, comparados com os demais países do leste europeu.

Entretanto, a crise de todo o sistema conduzido pela União Soviética cresce nos anos 80 e, como conseqüência, atinge fortemente a República Democrática Alemã. A progressiva insatisfação com questões políticas, econômicas, sociais e culturais vai provocar, a partir da cidade de Leipzig, uma série de acontecimentos que irão causar a queda do muro de Berlin em 09/11/1989 e, finalmente, a reunificação alemã em 03/10/1990. Nesta data, é extinta a República Democrática Alemã e a Constituição da República Federal da Alemanha passa a valer em todo o território oriental que passa a ter também o modelo de economia social de mercado e, por conseguinte, a integrar a União Européia.

$\mathrm{Na}$ época, ficou evidente o forte desequilíbrio entre a Alemanha ocidental e a oriental. Isto motivou a constituição urgente de políticas, programas e projetos para o desenvolvimento da parte oriental, com destaque para as questões de infraestrutura e de emprego. Como exemplo, é implantado (e que prossegue até hoje) um imposto adicional de solidariedade (Solitaritätszuschlag), cujos recursos são destinados a investimentos na parte oriental.

Portanto, a reunificação alemã revela, de imediato, o expressivo desequilíbrio entre a Alemanha Ocidental e a Alemanha Oriental. Por outro lado, é preciso reconhecer que, historicamente, sempre o território alemão ocidental foi bem mais industrializado que o território oriental, de base mais agrária e mercantil, o que exigiu um esforço adicional da antiga República Democrática Alemã visando à industrialização, logo após sua criação. Um dos exemplos mais significativos é a criação, em 1950, da nova cidade industrial de Eisenhüttenstadt, que significa cidade do aço, com uma grande usina siderúrgica estatal, hoje parte do grupo privado internacional Arcelor Mittal.

Agora, duas décadas após a reunificação da Alemanha, é importante avaliar o estado atual dos desequilíbrios alemães como resultado dos esforços realizados em busca de uma maior igualdade envolvendo toda a Alemanha no contexto das políticas de desenvolvimento regional da União Européia (UE). 


\section{DESEQUILÍBRIOS REGIONAIS NA ALEMANHA: 1995-2006}

Para o cálculo do índice de Williamson, que mede os desequilíbrios regionais, foram usadas as estatísticas fornecidas pelo EUROSTAT segundo diferentes níveis espaciais hierárquicos, NUTS, Nomenclatura das Unidades Territoriais para Fins Estatísticos, definidas institucionalmente pelos Estados-Membros da União Européia. Assim, cada país pode subdividir o seu território em três níveis regionais hierárquicos, por exemplo, em NUTS 1 que, por sua vez, é subdividido em NUTS 2 e este em NUTS 3.

A Alemanha é dividida primeiramente em 16 Länder, que correspondem ao nível NUTS 1 (Figura 1). Este nível mutatis mutandis pode ser comparado com os estados brasileiros. Em função das dificuldades de tradução da maioria dos nomes de lugares e regiões da Alemanha, optou-se por manter a grafia alemã.

O índice de Williamson foi calculado para toda a Alemanha reunificada, com base nestes 16 estados (Länder). O período analisado abrange os anos de 1995 a 2006, como foi explicado anteriormente. Ficou evidente que desde o primeiro ano da nossa análise, em 1995, o índice era baixo $(0,199)$ permanecendo praticamente o mesmo até $2006(0,198)$ (Tabela 1).

Portanto, neste período o impacto das transformações não consegue alterar, em termos relativos, os índices de desequilíbrios regionais da Alemanha dividida em estados (Länder), mesmo considerando que alguns são cidades-Estado, como Hamburg e Bremen, o que determina que os dados sobre PIB e PIB per capita sejam de caráter urbano e não regional. É preciso considerar que estes baixos índices, sobretudo o de 1995, cinco anos depois da unificação, não deixam de ser uma surpresa já que considerando as diferenças entre os territórios alemães do leste e do oeste seria para esperar índices maiores para este nível de análise. Como comparação, o Brasil dividido em estados tinha, em 2006, um índice de Williamson de 0,474.

Os desequilíbrios alemães são maiores dividindo o país em menores unidades espaciais, NUTS 2 segundo a UE, chamadas Regierungsbezirke na Alemanha, com um total de 39 regiões (Figura 2). Este fato, do crescimento do valor dos índices em menores unidades de análise, foi também constatado em outros países, como o Brasil (SILVA; SILVA; COELHO, 2006). Neste nível mais detalhado, que poderia ser comparado com as microrregiões brasileiras, a Alemanha continua praticamente com os mesmos índices, embora mais altos, no período analisado: 0,226, em 1995 e 0,227, em 2006 (Tabela 1). Como comparação, o Brasil, dividido em microrregiões, tinha um índice de 0,617, em 2006. A divisão em NUTS 2 permite dar mais destaque para a presença de regiões, várias de caráter urbano, com elevadíssimos PIB regional e PIB per capita, como Hamburg e Oberbayern que contrastam com muitas regiões com PIB regional e PIB per capita bem mais baixos, como Brandenburg-Nordost e Brandenburg-Südwest, na parte leste do país.

Já o último nível de agregação estatística, o NUTS 3, é chamado na Alemanha de Kreis. Observa-se que os desequilíbrios são bem maiores, portanto indicando valores muito próximos no decorrer dos anos (Tabela 1). Existem atualmente 429 NUTS 3 como consta na Figura 3. Este nível destaca ainda mais os contrastes entre áreas NUTS 3 muito ricos (em termos de PIB municipal e PIB per capita), como München Landkreis, e outros com os mesmos indicadores muito mais baixos, como Jerichower Land. A divisão em questão poderia ser comparada com os municípios brasileiros. O Brasil apresenta um índice neste nível de 0,881 em 2006. 


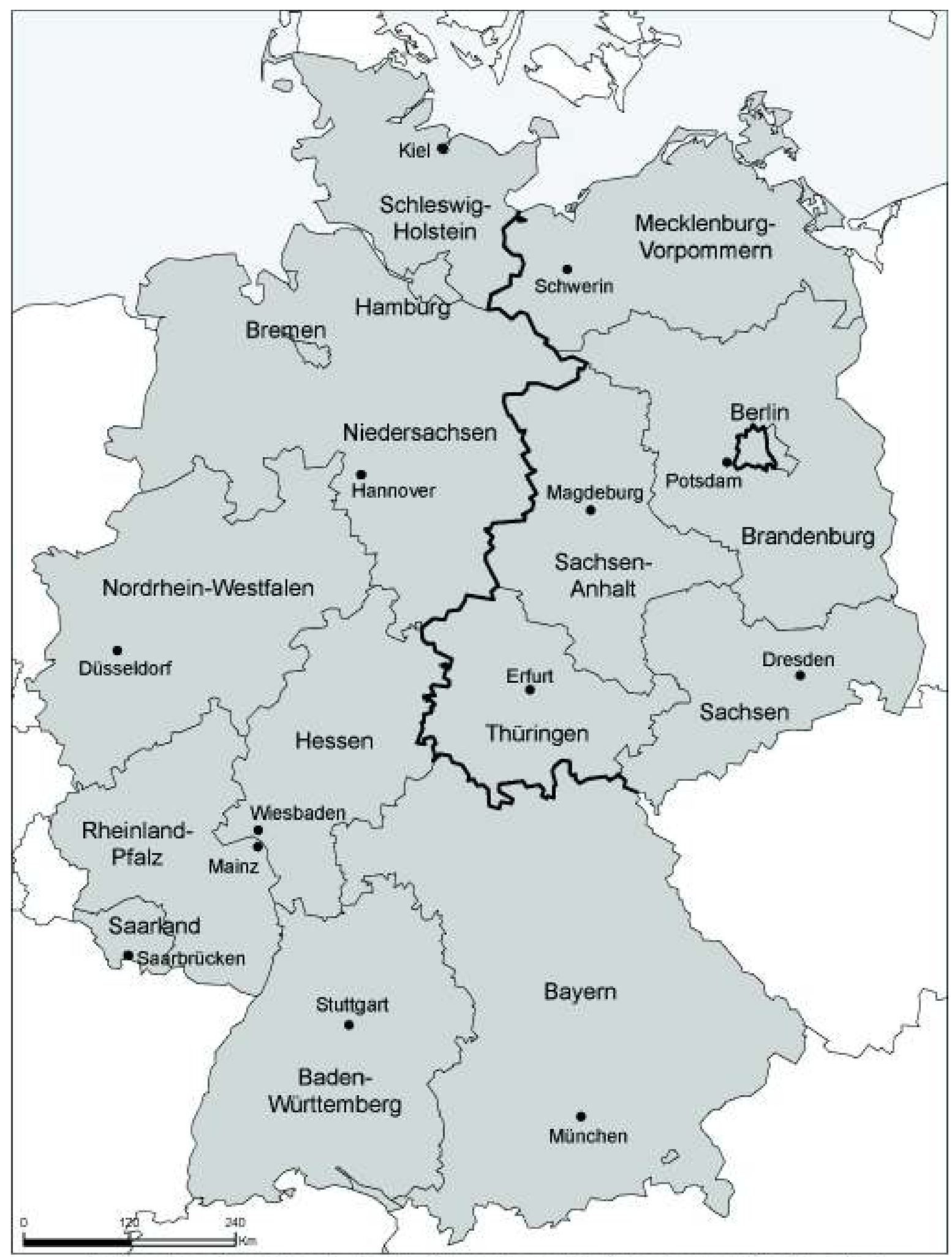

Limite entre a República Federal da Alemanha e a República Democrática Alemã até 03/10/1990

- Cidades

Figura 1 - Länder da República Federal da Alemanha - NUTS 1

Fonte : EUROSTAT. Administrative units - NUTS, 2006.

Elaboração: autoras. 
Tabela 1 - Alemanha - Índice de Williamson na base de NUTS 1, 2 e 3 - 1995-2006

\begin{tabular}{c|c|c|c}
\hline Ano & NUTS 1 & NUTS 2 & NUTS 3 \\
\hline 1995 & 0,199 & 0,226 & 0,404 \\
1996 & 0,196 & 0,225 & 0,406 \\
1997 & 0,197 & 0,226 & 0,411 \\
1998 & 0,199 & 0,229 & 0,413 \\
1999 & 0,198 & 0,229 & 0,420 \\
2000 & 0,200 & 0,231 & 0,421 \\
2001 & 0,204 & 0,235 & 0,427 \\
2002 & 0,201 & 0,231 & 0,419 \\
2003 & 0,199 & 0,231 & 0,421 \\
2004 & 0,196 & 0,227 & 0,416 \\
2005 & 0,197 & 0,227 & 0,417 \\
2006 & 0,198 & 0,227 & 0,416 \\
\hline
\end{tabular}

Fonte: EUROSTAT. Gross domestic product at NUTS 1, 2 e 3, 1995 a 2006; Population on 1. January of each year at NUTS 1 e 2, 1996 a 2007; Annual average population at NUTS 3, 1995 a 2006. Elaboração: autoras.

Por outro lado, mantendo a divisão da Alemanha, para efeitos comparativos, entre a parte ocidental e a parte oriental, a Tabela 2 permite comparar os desequilíbrios regionais, entre 1995 e 2006, com base nas três divisões regionais já apresentadas (NUTS 1, NUTS 2 e NUTS 3).

Tabela 2 - Alemanha Ocidental e Oriental - Índice de Williamson na base de NUTS 1, 2 e 3 - 1995-2006

\begin{tabular}{lcccccc}
\hline Ano & \multicolumn{3}{c}{ Alemanha Ocidental } & \multicolumn{3}{c|}{ Alemanha Oriental } \\
& & & & & \\
& NUTS 1 & NUTS 2 & NUTS 3 & NUTS 1 & NUTS2 & NUTS 3 \\
1995 & 0,126 & 0,167 & 0,382 & 0,203 & 0,207 & 0,295 \\
1996 & 0,132 & 0,175 & 0,387 & 0,177 & 0,182 & 0,293 \\
1997 & 0,135 & 0,178 & 0,393 & 0,162 & 0,166 & 0,287 \\
1998 & 0,136 & 0,180 & 0,394 & 0,155 & 0,157 & 0,286 \\
1999 & 0,138 & 0,183 & 0,404 & 0,143 & 0,147 & 0,270 \\
2000 & 0,139 & 0,185 & 0,405 & 0,137 & 0,140 & 0,260 \\
2001 & 0,149 & 0,194 & 0,413 & 0,123 & 0,125 & 0,256 \\
2002 & 0,152 & 0,194 & 0,408 & 0,107 & 0,110 & 0,246 \\
2003 & 0,153 & 0,196 & 0,411 & 0,096 & 0,100 & 0,251 \\
2004 & 0,151 & 0,193 & 0,406 & 0,079 & 0,086 & 0,247 \\
2005 & 0,152 & 0,193 & 0,407 & 0,080 & 0,087 & 0,243 \\
2006 & 0,153 & 0,192 & 0,406 & 0,073 & 0,078 & 0,239 \\
\hline
\end{tabular}

Fonte: EUROSTAT. Gross domestic product at NUTS 1, 2 e 3, 1995 a 2006; Population on 1. January of each year at NUTS 1 e 2, 1996 a 2007; Annual average population at NUTS 3, 1995 a 2006. Elaboração: autoras.

Considerando o nível NUTS 1, verifica-se que os índices na Alemanha Oriental foram mais altos entre 1995 e 1999 do que na Alemanha Ocidental mas diminuíram bastante nos últimos sete anos do período analisado, atingindo valores mais baixos que a parte ocidental da Alemanha. Quanto ao nível NUTS 2, somente nos anos 1995 e 1996, os índices foram mais altos na Alemanha Oriental, diminuindo significativamente até 2006. A análise, na base de NUTS 3, aponta que, em todos os anos, desde 1995, os índices são menores na parte oriental e diminuindo ainda constantemente até 2006, contrastando com o aumento na parte ocidental. 


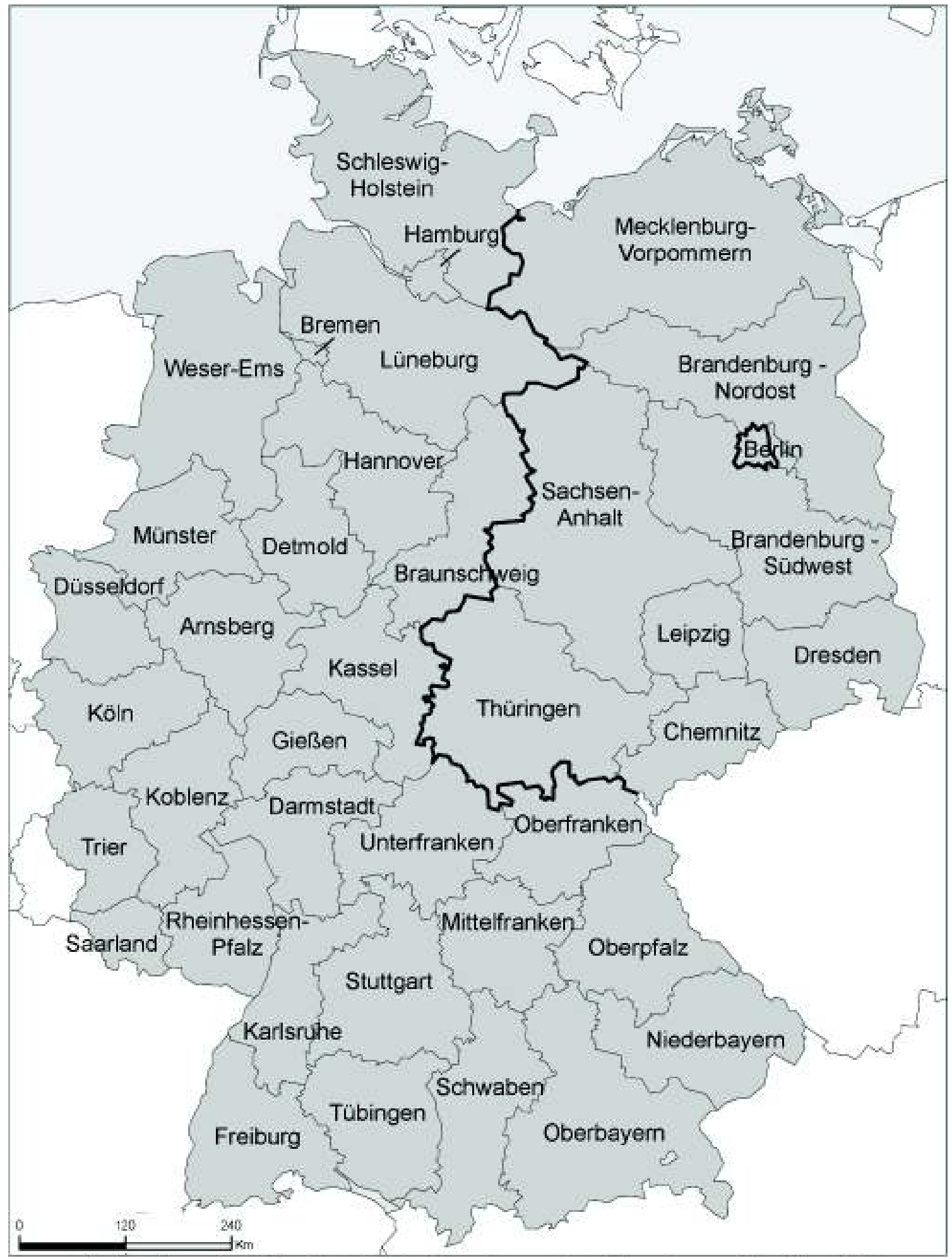

Limite entre a República Federal da Alemanha e a República Democrática Alemă até 03/10/1990

Figura 2: Regierungsbezirke da República Federal da Alemanha - NUTS 2

Fonte: EUROSTAT. Administrative units - NUTS, 2006.

Elaboração: autoras. 


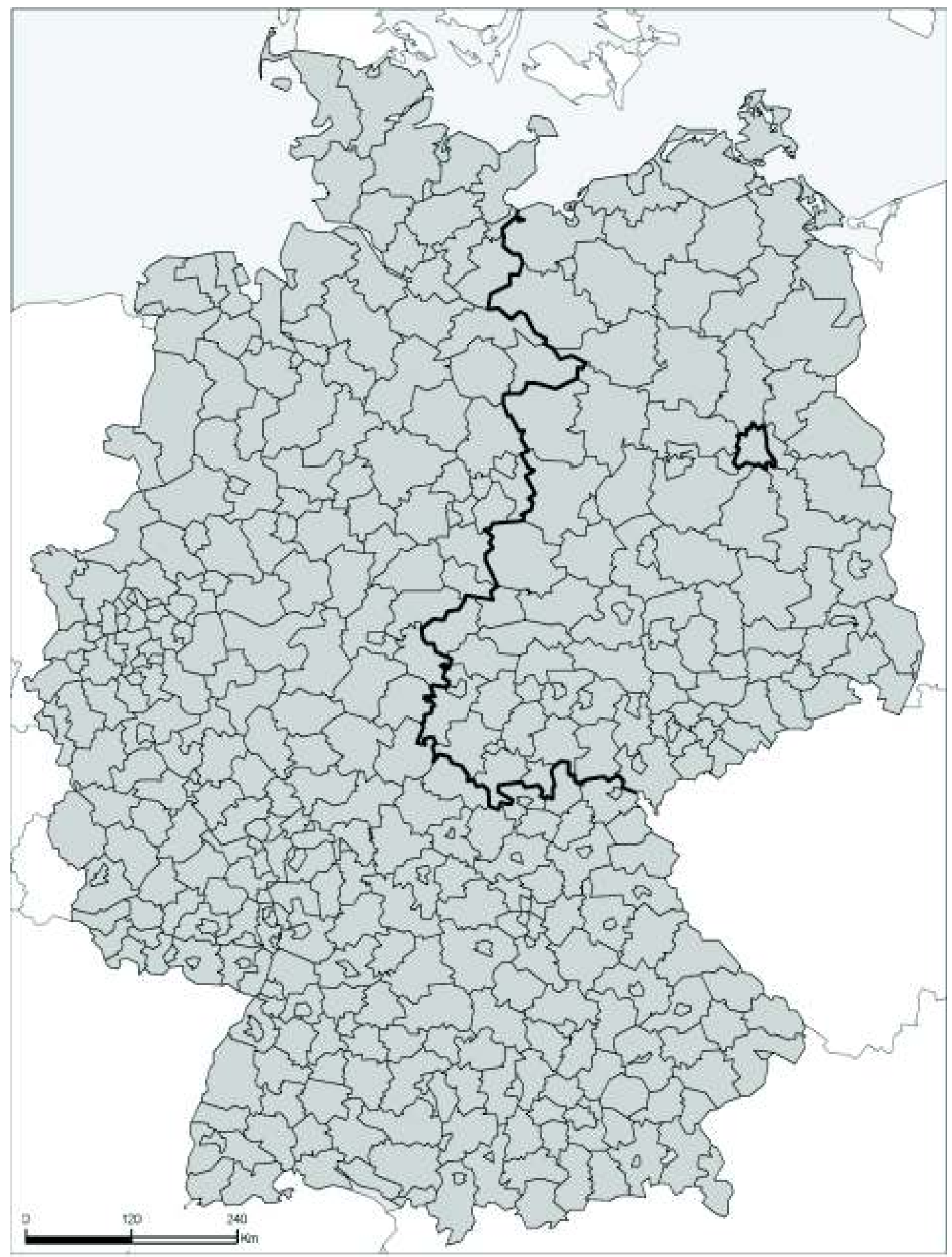

Limite entre a República Federal da Alemanha e a República Democrática Alemã até $03 / 10 / 1990$

Figura 3: Kreise da República Federal da Alemanha - NUTS 3

Fonte das informações: EUROSTAT. Administrative units - NUTS, 2006. 
Na parte ocidental da Alemanha, os índices aumentam no período analisado em todos os níveis de análise (NUTS 1, NUTS 2, NUTS 3) e, de forma bastante expressiva, eles diminuem na parte oriental. É significativo observar que, em 1995, apenas cinco anos após a reunificação, os desequilíbrios na Alemanha Oriental eram maiores para os territórios NUTS 1 e NUTS 2 do que na Alemanha Ocidental enquanto que o nível NUTS 3 era maior no oeste. Em 2006, 16 anos após a reunificação, a antiga Alemanha Oriental tem indicadores inferiores aos da Alemanha Ocidental em todas as escalas territoriais. Isto permite afirmar que os impactos da reunificação para o leste da Alemanha foram bastante favoráveis. Ou seja, os desequilíbrios regionais foram reduzidos nesta área enquanto que na parte ocidental eles cresceram.

As comparações ficam claramente evidenciadas sob a forma gráfica. A Figura 4 visualiza a distribuição dos índices de Williamson na base de NUTS 1 (os mais baixos valores), NUTS 2 e NUTS 3 (os mais altos valores), para toda a Alemanha.

As formas da Figura 4 praticamente se repetem quando os desequilíbrios são analisados tomando como base somente o lado ocidental da Alemanha (Figura 5).

Já o território oriental apresenta valores muito próximos nos níveis NUTS 1 e 2, com forte redução no período. A redução no nível NUTS 3 é também significativo (Figura 6).

\section{DESEQUILÍBRIOS DE RENDA PER CAPITA NA ALEMANHA: UM DESTAQUE NECESSÁRIO}

Como foi mencionado, a renda per capita faz parte do índice de Williamson, comparada com dados sobre PIB total e população. É importante, entretanto, realizar uma análise em separado da renda per capita considerando que a mesma é reconhecida, preliminarmente, como uma relevante medida geral, reveladora da distribuição de renda em unidades territoriais escolhidas. Quanto maior o nível escalar da pesquisa, ou seja, quanto menor a dimensão espacial do território, mais significativos serão os resultados.

Assim, a Tabela 3 mostra a evolução da renda per capita da parte ocidental e oriental da Alemanha entre 1995 e 2006.

Tabela 3: Comparação entre a renda per capita da Alemanha Ocidental e da Alemanha Oriental - 1995-2006

\begin{tabular}{|c|c|c|c|}
\hline Ano & $\begin{array}{c}\text { Renda per capita }(\mathrm{em} €) \\
\text { Alemanha Ocidental }\end{array}$ & $\begin{array}{c}\text { Renda per capita (em } € \text { ) } \\
\text { Alemanha } \\
\text { Oriental }\end{array}$ & \% Oriental/Ocidental \\
\hline 1995 & 25.366 & 17.094 & 67,39 \\
\hline 1996 & 25.124 & 17.232 & 68,59 \\
\hline 1997 & 24.924 & 17.046 & 68,39 \\
\hline 1998 & 25.553 & 17.275 & 67,61 \\
\hline 1999 & 26.262 & 17.851 & 67,97 \\
\hline 2000 & 26.904 & 18.162 & 67,51 \\
\hline 2001 & 27.465 & 18.640 & 67,87 \\
\hline 2002 & 27.736 & 19.149 & 69,04 \\
\hline 2003 & 27.965 & 19.441 & 69,52 \\
\hline 2004 & 28.563 & 19.910 & 69,70 \\
\hline 2005 & 29.006 & 20.165 & 69,52 \\
\hline 2006 & 30.073 & 20.825 & 69,25 \\
\hline
\end{tabular}

Fonte: EUROSTAT. Gross domestic product (Millions of euros) at current market prices 1995 a 2006; Population on 1. January of each year 1996 a 2007. Elaboração: autoras.

Percebe-se imediatamente que a renda per capita da antiga Alemanha Oriental é bem inferior a da Alemanha Ocidental e que não houve uma modificação significativa de 1995 a 2006 (12 anos).

Mercator - volume 9, número 20, 2010: set./dez. 


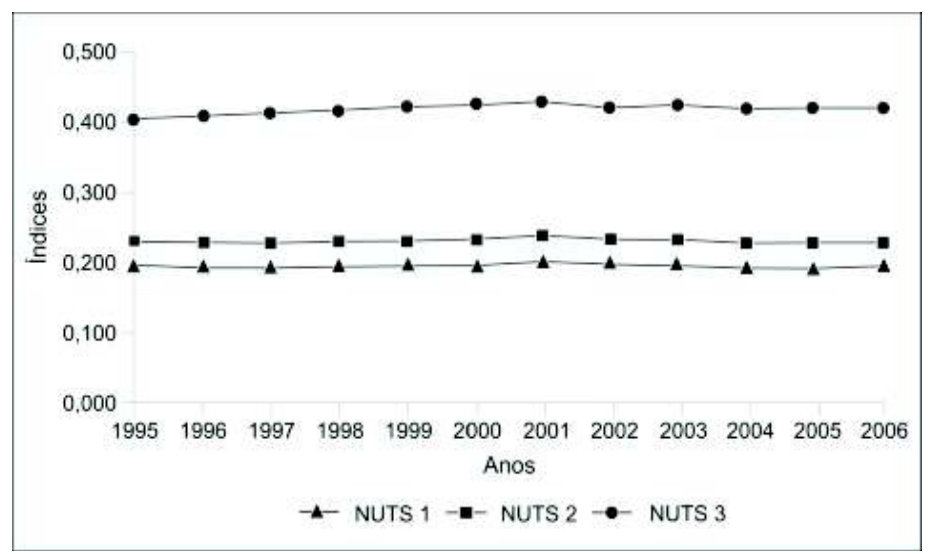

Figura 4 - Alemanha - Índice de Williamson na base de NUTS 1, 2 e 3 - 1995-2006

Fonte: EUROSTAT. Gross domestic product at NUTS 1, 2 e 3, 1995 a 2006; Population on 1. January of each year at NUTS 1 e 2, 1996 a 2007; Annual average population at NUTS 3, 1995 a 2006.

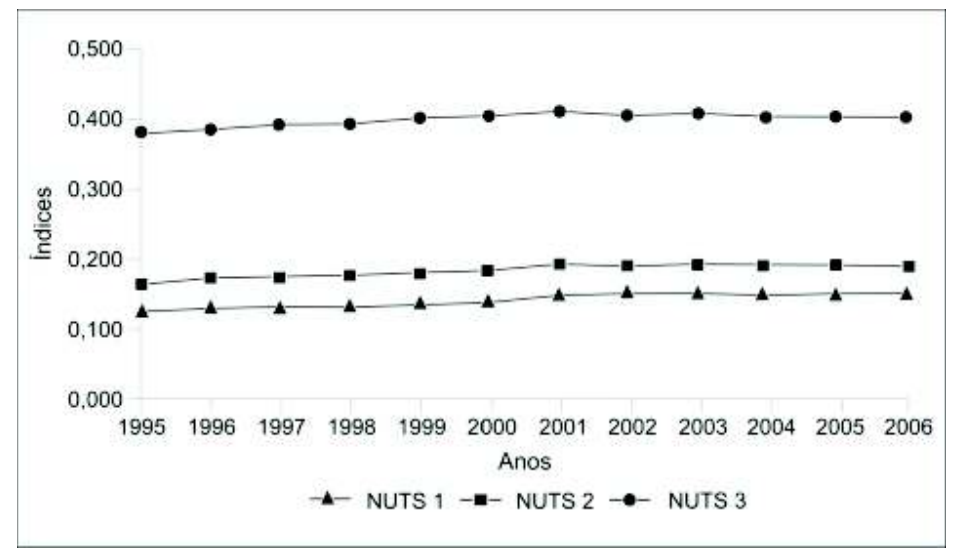

Figura 5 - Alemanha Ocidental - Índice de Williamson na base de NUTS 1, 2 e 3 - 1995-2006

Fonte: EUROSTAT. Gross domestic product at NUTS 1, 2 e 3, 1995 a 2006; Population on 1. January of each year at NUTS 1 e 2, 1996 a 2007; Annual average population at NUTS 3, 1995 a 2006.

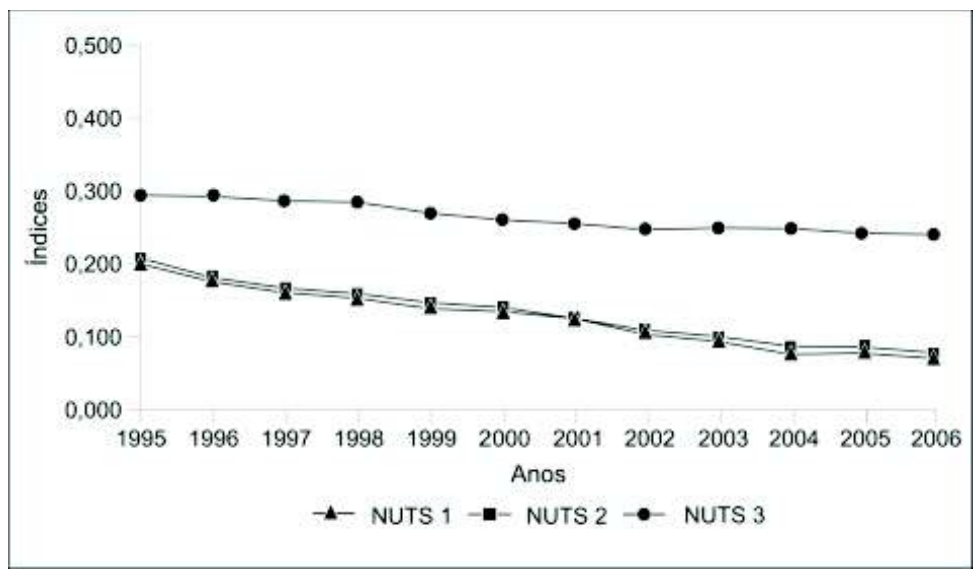

Figura 6 - Alemanha Oriental - Índice de Williamson na base de NUTS 1, 2 e 3 - 1995-2006

Fonte: EUROSTAT. Gross domestic product at NUTS 1, 2 e 3, 1995 a 2006; Population on 1. January of each year at NUTS 1 e 2, 1996 a 2007; Annual average population at NUTS 3, 1995 a 2006. Elaboração: autoras. 
Com efeito, o PIB per capita dos alemães orientais representava $67,39 \%$ da renda per capita dos alemães ocidentais e isto evoluiu, em 2006, para apenas 69,25\%.

Os desequilíbrios são bem maiores quando se detalha mais as comparações usando as escalas territoriais NUTS 1, NUTS 2 e NUTS 3.

A Tabela 4 apresenta, para as regiões NUTS 1, os maiores e os menores PIB per capita para a Alemanha unificada, a Alemanha Ocidental e a Alemanha Oriental. Foram destacadas, na tabela, as unidades territoriais que são cidades (cidade-Estado, como é o caso de Hamburg e Bremen, e a cidade-Capital, Berlin), visando tornar mais justa a comparação entre os NUTS 1. Portanto, para o conjunto da Alemanha, não é recomendável comparar as cidades-Estado de Hamburg e Bremen, cada uma definida como NUTS 1, com as regiões, também NUTS 1, de Hessen, Thüringen, Mecklenburg-Vorpommern, que entre si podem ser perfeitamente comparáveis. Ou seja, deve haver compatibilidade nas escalas de análise. Assim, a região de Mecklenburg-Vorpommern, na parte oriental, representava, em 2006, uma renda per capita equivalente a 56,0\% da renda per capita da região de Hessen, na Alemanha Ocidental. Em 1995, esta relação foi menor (51,6\%), comparando Thüringen e Hessen. Por outro lado constata-se que o lado oriental da Alemanha é mais equilibrado que o ocidental, isto é, revela uma bem menor variação entre o mais alto e o mais baixo valor de PIB per capita.

Tabela 4 - PIB per capita da Alemanha NUTS 1 - 1995-2006 (em €)

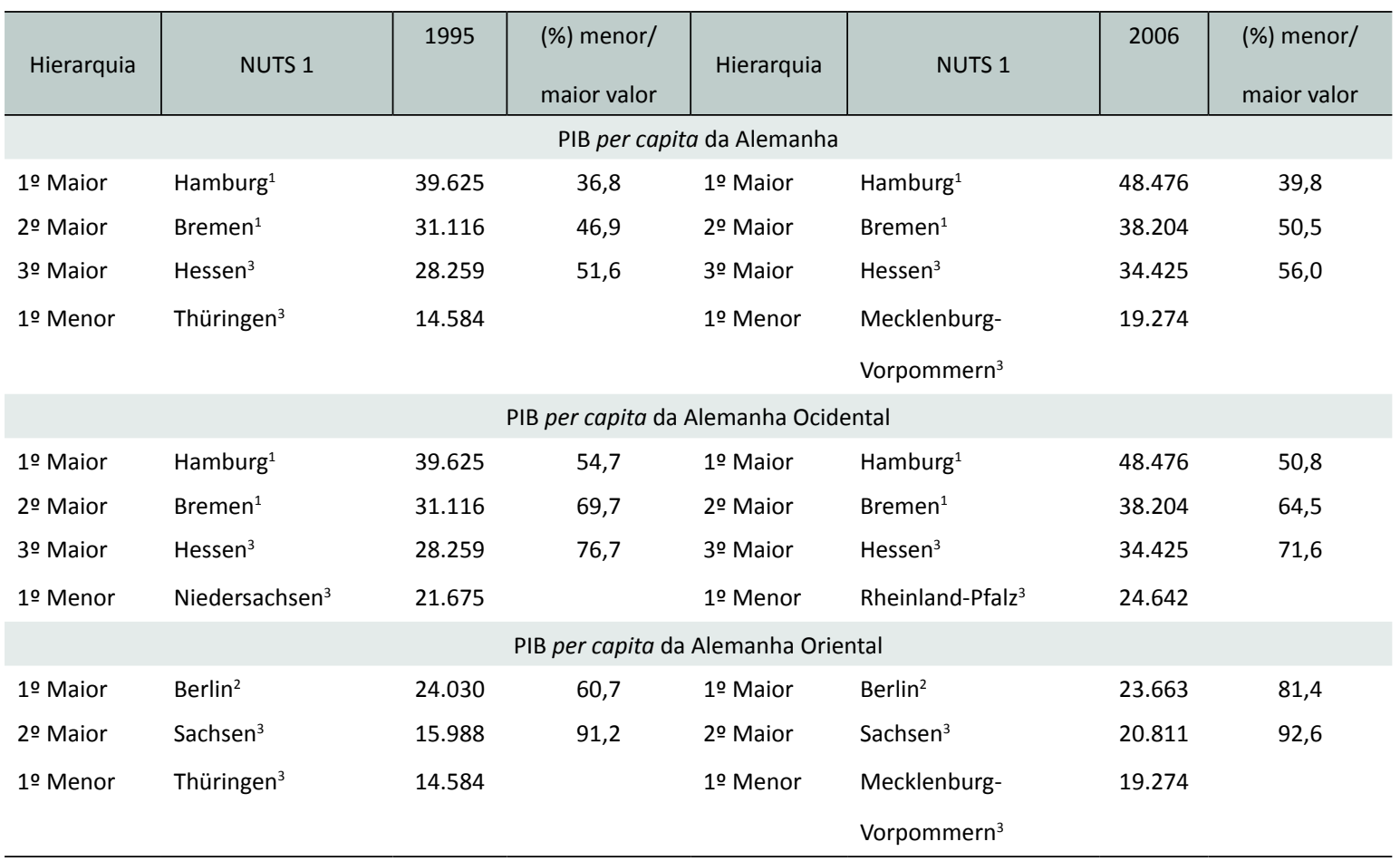

${ }^{1}$ Cidade-Estado. ${ }^{2}$ Cidade-Capital. ${ }^{3}$ Tomado como referência para o cálculo, menor valor/maior valor.

Fonte: EUROSTAT. Gross domestic product (Millions of euros) at current market prices 1995-2006; Population on 1. January of each year 1996-2007. Elaboração: autoras.

A Tabela 5 faz a mesma comparação para as regiões NUTS 2. Além das cidades, é citado o caso do estado de Thüringen, (NUTS 1), que não foi subdividido em NUTS 2 e 3, ocorrendo o mesmo com as cidades de Hamburg e Berlin, o que indica que a comparação mais significativa para toda a Alemanha é entre Brandenburg-Nordost e Oberbayern, como consta na tabela. Em 2006, a menor renda per capita, a de Brandenburg-Nordost representava apenas 45,3\% da região Oberbayern, na parte oeste, um pouco abaixo do valor registrado em 1995 (46,5\%). Também neste nível territorial, 
a parte oriental é mais equilibrada que a ocidental, ou seja, existe mais aproximação entre o menor e o maior valor de PIB per capita, mas deve ser destacado que todos os valores mínimos e máximos são bem abaixo dos da Alemanha Ocidental.

Tabela 5 - PIB per capita da Alemanha NUTS 2 - 1995-2006 (em €)

\begin{tabular}{|c|c|c|c|c|c|c|c|}
\hline Hierarquia & NUTS 2 & 1995 & $\begin{array}{l}\text { (\%) menor/ } \\
\text { maior valor }\end{array}$ & Hierarquia & NUTS 2 & 2006 & $\begin{array}{l}\text { (\%) menor/ } \\
\text { maior valor }\end{array}$ \\
\hline \multicolumn{8}{|c|}{ PIB per capita da Alemanha } \\
\hline 1을 Maior & Hamburg $^{1}$ & 39.625 & 37,0 & 10 Maior & Hamburg $^{1}$ & 48.476 & 38,0 \\
\hline 2ㅇ Maior & Darmstadt $^{3}$ & 32.199 & 45,5 & 20 Maior & Oberbayern ${ }^{5}$ & 40.697 & 45,3 \\
\hline 3으aior & Oberbayern ${ }^{5}$ & 31.558 & 46,5 & 1을 Menor & Brandenburg - & 18.438 & \\
\hline 10 Menor & Thüringen ${ }^{4}$ & 14.584 & & & Nordost $^{5}$ & & \\
\hline \multirow[t]{2}{*}{ 2을 Menor } & Brandenburg - & 14.662 & & & & & \\
\hline & Nordost $^{5}$ & & & & & & \\
\hline \multicolumn{8}{|c|}{ PIB per capita da Alemanha Ocidental } \\
\hline 1을 Maior & Hamburg $^{1}$ & 39.625 & 47,6 & 10 Maior & Hamburg $^{1}$ & 48.476 & 42,2 \\
\hline 2 Maior & Darmstadt $^{3}$ & 32.199 & 58,6 & 20 Maior & Oberbayern $^{5}$ & 40.697 & 50,3 \\
\hline 3o Maior & Oberbayern ${ }^{5}$ & 31.558 & 59,8 & 1을 Menor & Lüneburg 5 & 20.474 & \\
\hline 10 Menor & Lüneburg $^{5}$ & 18.863 & & & & & \\
\hline \multicolumn{8}{|c|}{ PIB per capita da Alemanha Oriental } \\
\hline 10 Maior & Berlin² & 24.030 & 61,0 & 1ำ Maior & Berlin² & 23.663 & 77,9 \\
\hline 2o Maior & Leipzig $^{3}$ & 17.729 & 82,7 & 2ㅇ Maior & Leipzig $^{3}$ & 21.377 & 86,2 \\
\hline \multirow[t]{2}{*}{ 3 Maior } & Brandenburg - & 16.505 & 88,8 & 3으aior & Dresden $^{3}$ & 21.313 & 86,5 \\
\hline & Südwest ${ }^{5}$ & & & 4을 Maior & Brandenburg - & 20.746 & 88,9 \\
\hline 1을 Menor & Thüringen ${ }^{4}$ & 14.584 & & & Südwest ${ }^{5}$ & & \\
\hline \multirow[t]{3}{*}{ 2ㅇ Menor } & Brandenburg - & 14.662 & & & & & \\
\hline & Nordost $^{5}$ & & & 1을 Menor & Brandenburg - & 18.438 & \\
\hline & & & & & Nordost $^{5}$ & & \\
\hline
\end{tabular}

${ }^{1}$ Cidade-Estado. ${ }^{2}$ Cidade-Capital. ${ }^{3}$ Cidade. ${ }^{4}$ Estado sem subdivisão em NUTS 2.

${ }^{5}$ Tomado como referência para o cálculo, menor valor/maior valor.

Fonte: EUROSTAT. Gross domestic product (Millions of euros) at current market prices 1995-2006; Population on 1. January of each year 1996-2007. Elaboração: autoras.

Encerrando a comparação, os valores para as regiões NUTS 3 para toda a Alemanha são apresentados na Tabela 6. Em 2006, a área de Jerichower Land, na parte leste, tinha uma renda per capita que equivalia a somente $11,8 \%$ da renda per capita de München Landkreis, na parte oeste. Comparando com 1995 (15,0\%), percebe-se que a realidade piorou. Nesta escala territorial estão presentes fortes desequilíbrios, particularmente na Alemanha Ocidental.

Assim, comparando os três níveis de subdivisão para toda a Alemanha, só ocorreu uma melhoria da relação entre a região com mais alto e mais baixo PIB per capita no nível NUTS 1. Na Alemanha Oriental, com baixos valores de PIB e PIB per capita, registrou-se uma pequena melhoria nos três níveis de análise, acontecendo o contrário na Alemanha Ocidental onde a amplitude entre regiões com maior e menor valor aumentou. 
Tabela 6 - PIB per capita da Alemanha NUTS 3 - 1995-2006 (em €)

\begin{tabular}{|c|c|c|c|c|c|c|c|}
\hline Hierarquia & NUTS 3 & 1995 & $\begin{array}{l}\text { (\%) menor/ } \\
\text { maior valor }\end{array}$ & Hierarquia & NUTS 3 & 2006 & $\begin{array}{l}\text { (\%) menor/ } \\
\text { maior valor }\end{array}$ \\
\hline \multicolumn{8}{|c|}{ PIB per capita da Alemanha } \\
\hline 1을 Maior & $\begin{array}{l}\text { Frankfurt am Main, } \\
\text { Kreisfreie Stadt }^{1}\end{array}$ & 63.774 & 11,9 & 1음oior & $\begin{array}{l}\text { München, } \\
\text { Landkreis }^{2}\end{array}$ & 90.497 & 11,8 \\
\hline 2 Maior & $\begin{array}{l}\text { Düsseldorf, } \\
{\text { Kreisfreie Stadt }{ }^{1}}\end{array}$ & 56.597 & 13,4 & 10 Menor & $\begin{array}{l}\text { Jerichower } \\
\text { Land }^{2}\end{array}$ & 10.713 & \\
\hline 3o Maior & Ludwigshafen am & 54.491 & 14,0 & & & & \\
\hline & $\begin{array}{l}\text { Rhein, Kreisfreie } \\
\text { Stadt }^{1}\end{array}$ & & & & & & \\
\hline 4을 Maior & Regensburg, & 51.627 & 14,7 & & & & \\
\hline & Kreisfreie Stadt ${ }^{1}$ & & & & & & \\
\hline 5o Maior & München, Landkreis² & 50.570 & 15,0 & & & & \\
\hline 1을 Menor & Jerichower Land² & 7.608 & & & & & \\
\hline \multicolumn{8}{|c|}{ PIB per capita da Alemanha Ocidental } \\
\hline 1음or & $\begin{array}{l}\text { Frankfurt am Main, } \\
\text { Kreisfreie Stadt }^{1}\end{array}$ & 63.774 & 17,1 & 1o Maior & $\begin{array}{l}\text { München, } \\
\text { Landkreis }^{2}\end{array}$ & 90.497 & 13,8 \\
\hline 2음oior & $\begin{array}{l}\text { Düsseldorf, } \\
\text { Kreisfreie Stadt }^{1}\end{array}$ & 56.597 & 19,3 & 10 Menor & Südwestpfalz² & 12.515 & \\
\hline 3o Maior & $\begin{array}{l}\text { Ludwigshafen am } \\
\text { Rhein, Kreisfreie } \\
\text { Stadt }^{1}\end{array}$ & 54.491 & 20,0 & & & & \\
\hline 4o Maior & $\begin{array}{l}\text { Regensburg, } \\
\text { Kreisfreie Stadt }^{1}\end{array}$ & 51.627 & 21,1 & & & & \\
\hline 5o Maior & München, Landkreis² & 50.570 & 21,6 & & & & \\
\hline 1을 Menor & Südwestpfalz ${ }^{2}$ & 10.915 & & & & & \\
\hline \multicolumn{8}{|c|}{ PIB per capita da Alemanha Oriental } \\
\hline 1o Maior & $\mathrm{Harz}^{2}$ & 32.932 & 23,1 & 1o Maior & $\operatorname{Harz}^{2}$ & 45.835 & 23,4 \\
\hline 1을 Menor & Jerichower Land ${ }^{2}$ & 7.608 & & 10 Menor & $\begin{array}{l}\text { Jerichower } \\
\text { Land }^{2}\end{array}$ & 10.713 & \\
\hline
\end{tabular}

${ }^{1}$ Cidade. ${ }^{2}$ Tomado como referência para o cálculo, menor valor/maior valor.

Fonte: EUROSTAT. Gross domestic product (Millions of euros) at current market prices 1995-2006; Population on 1. January of each year 1996-2007. Elaboração: autoras.

\section{CONSIDERAÇÕES FINAIS}

A análise permite afirmar que os desequilíbrios regionais na Alemanha pós-reunificação são e foram recentemente relativamente reduzidos, mas que é preciso percorrer ainda um caminho para se obter resultados mais expressivos.

Com efeito, os índices gerais de desequilíbrios regionais alemães para os Länder, (NUTS 1) e Bezirke (NUTS 2) são baixos no período analisado e permanecem praticamente os mesmos. Para os Kreise (NUTS 3), os índices são mais altos e apresentam um pequeno aumento. Quando se analisam os índices para a parte oriental e para a parte ocidental, percebe-se que o território da ex-República Democrática Alemã mostra menos desequilíbrios e que ocorre significativa redução nos seus índices.

Mercator - volume 9, número 20, 2010: set./dez. 
Os desequilíbrios ficam mais expressivos na análise em separado da renda per capita tanto para NUTS 1 como, e sobretudo, para NUTS 2 e NUTS 3. É possível também afirmar, analisando os PIB totais e os PIB per capita, que o leste alemão é mais homogêneo enquanto que o oeste é mais heterogêneo já que existem unidades territoriais com PIB total e PIB per capita muito elevados e outros mais baixos.

Em síntese, são estes os desequilíbrios que são mais diretamente percebidos pelos habitantes do leste alemão em comparação com os do oeste. Como exemplo, os salários industriais para as mesmas funções são mais baixos na parte oriental do que na ocidental, como foi possível para os autores verificar in loco em uma moderna fábrica alemã de automóveis em Dresden/Sachsen, na parte oriental (julho 2009), comparada com outra fábrica do mesmo grupo na parte oeste da Alemanha. Esta situação reproduz exatamente o que acontece no Brasil, por exemplo, entre a indústria automobilística do Nordeste e a do Sudeste.

Portanto, a Alemanha tem ainda um grande desafio político, econômico e social, o de reduzir seus desequilíbrios regionais em todas as escalas do seu território, especialmente, no que diz respeito à renda per capita.

\section{REFERÊNCIA BIBLIOGRÁFICA}

EUROSTAT. Administrative units - NUTS, 2006. Disponível em: $<$ http://epp.eurostat.ec. europa.eu/portal/ page/portal/gisco/geodata/reference. Acesso em: 22 nov. 2009.

EUROSTAT. Population by sex and age on 1. January of each year, 1996 a 2007. Disponível em: $<$ http:// epp.eurostat.ec.europa.eu/extraction/evalight/EVAlight.jsp?A=1\&language $=$ en\&root $=/$ theme $3 / \mathrm{demo} /$ demo_pjan>. Acesso em: 07 mar. 2009.

EUROSTAT. Population at 1st January by sex and age from 1990 onwards at NUTS 1 and 2 , 1996 a 2007. Disponível em: <http://epp.eurostat.ec.europa.eu/extraction/evalight/EVAlight.jsp? $\mathrm{A}=1$ \&language $=$ en\&root $=/$ theme $1 / \mathrm{reg} / \mathrm{reg}$ d $2 \mathrm{ja}>$. Acesso em: $04 \mathrm{mar} .2009$.

EUROSTAT. Annual average population by sex at NUTS 3, 1995 a 2006. Disponível em: <http://epp. eurostat.ec.europa.eu/extraction/evalight/EVAlight.jsp?A=1\&language=en\&root=/theme1/reg/reg_d3avg $>$. Acesso em: 04 mar. 2009.

EUROSTAT. Gross domestic product (Millions of euro) at current market prices 1995 a 2006. Disponível $\mathrm{em}:<\mathrm{http}: / /$ epp.eurostat.ec.europa.eu/extraction/evalight/EVAlight.jsp?A=1\&langua ge=en\&root=/theme2/ nama/nama_gdp_c $>$. Acesso em: 07 mar. 2009.

EUROSTAT. Gross domestic product (millions of euro) at current market prices at NUTS 1, 2 and 31995 a 2006. Disponível em: <http://epp.eurostat.ec.europa.eu/extraction/evalight/ EVAlight. jsp?A=1\&language=en\&root=/theme1/reg/reg_e3gdp $>$. Acesso em: 04 mar. 2009.

SCHMID, G. F. Kleine Deutschlandkunde. 2. ed. Stuttgart: Ernst Klett, 1996.

SILVA, S. B. de M. e. Nova Alemanha, Ano II. Cadernos de Geociências, Salvador: GEO, UFBA, v. 1, n. 3, p.13-16, dez. 1992.

SILVA, S. B. de M. e; SILVA, B. C. N.; COELHO, A. S. Desequilíbrios e desigualdades regionais no Brasil e nos estados brasileiros. João Pessoa: Ed. Grafset, 2008.

WILLIAMSON, J. G. Desigualdade regional e o processo de desenvolvimento nacional: descrição de padrões. In: SCHWARTZMAN, J. (Org.). Economia Regional. Textos escolhidos. Belo Horizonte: Cedeplar, 1977. p. 53-116.

Trabalho enviado em setembro de 2010

Trabalho aceito em novembro de 2010 